\section{Sequence-specific 2'-0-methoxyethyl antisense oligonucleotides activate human platelets through glycoprotein VI, triggering formation of platelet-leukocyte aggregates}

\begin{abstract}
Martina H. Lundberg Slingsby, ${ }^{1,2}$ Prakrith Vijey, ${ }^{2}$ I-Ting Tsai, ${ }^{1,2}$ Harvey Roweth, ${ }^{2}$ Genevieve Couldwell, ${ }^{2}$ Adrian R. Wilkie, ${ }^{1,2}$ Hans Gaus, ${ }^{3}$ Jazana M. Goolsby, ${ }^{2}$ Ross Okazaki, ${ }^{2}$ Brooke E. Terkovich, ${ }^{2}$ John W. Semple, ${ }^{4,5}$ Jonathan N. Thon, ${ }^{2}$ Scott P. Henry, ${ }^{3}$ Padmakumar Narayanan ${ }^{3}$ and Joseph E. Italiano Jr. ${ }^{1,2}$

${ }^{1}$ Vascular Biology Program, Department of Surgery, Boston Children's Hospital, Boston, MA, USA; ${ }^{2}$ Division of Hematology, Brigham and Women's Hospital, Harvard Medical School, Boston, MA, USA; ${ }^{3}$ Nonclinical Development, Ionis Pharmaceuticals Inc.,

Carlsbad, CA, USA; ${ }^{4}$ Departments of Pharmacology and Medicine, University of Toronto, Toronto, Ontario, Canada and ${ }^{5}$ Division of Hematology and Transfusion Medicine, Lund University, Lund, Sweden
\end{abstract}

\section{ABSTRACT}

A ntisense oligonucleotides (ASO) are DNA-based, disease-modifying drugs. Clinical trials with 2'-O-methoxyethyl (2'MOE) ASO have shown dose- and sequence-specific lowering of platelet counts according to two phenotypes. Phenotype 1 is a moderate (but not clinically severe) drop in platelet count. Phenotype 2 is rare, severe thrombocytopenia. This article focuses on the underlying cause of the more common phenotype 1, investigating the effects of ASO on platelet production and platelet function. Five phosphorothioate ASO were studied: three 2'MOE sequences; 487660 (no effects on platelet count), 104838 (associated with phenotype 1), and 501861 (effects unknown) and two CpG sequences; 120704 and ODN 2395 (known to activate platelets). Human cord bloodderived megakaryocytes were treated with these ASO to study their effects on proplatelet production. Platelet activation (determined by surface Pselectin) and platelet-leukocyte aggregates were analyzed in ASO-treated blood from healthy human volunteers. None of the ASO inhibited proplatelet production by human megakaryocytes. All the ASO were shown to bind to the platelet receptor glycoprotein VI $\left(\mathrm{K}_{\mathrm{D}} \sim 0.2-1.5 \mu \mathrm{M}\right)$. CpG ASO had the highest affinity to glycoprotein VI, the most potent platelet-activating effects and led to the greatest formation of platelet-leukocyte aggregates. 2'MOE ASO 487660 had no detectable platelet effects, while 2'MOE ASOs 104838 and 501861 triggered moderate platelet activation and SYKdependent formation of platelet-leukocyte aggregates. Donors with higher platelet glycoprotein VI levels had greater ASO-induced platelet activation. Sequence-dependent ASO-induced platelet activation and platelet-leukocyte aggregates may explain phenotype 1 (moderate drops in platelet count). Platelet glycoprotein VI levels could be useful as a screening tool to identify patients at higher risk of ASO-induced platelet side effects.

\section{Introduction}

Antisense oligonucleotides (ASO) are short, synthetic, single-stranded DNA molecules (between 8 and 50 nucleotides) that bind to specific mRNA segments through Watson-Crick base pairing. ${ }^{1}$ They are designed to modulate targeted mRNA by interfering with its function or promoting its degradation, ultimately altering protein expression. ${ }^{1,2}$ ASO offer therapeutic opportunities for treating rare genetic diseases such as spinal muscular atrophy (nusinersen), ${ }^{3,4}$ hereditary transthyretin amyloidosis (inotersen) ${ }^{5}$ and homozygous familial hypercholesterolemia (mipomersen). ${ }^{6}$ There has been a surge in ASO entering clinical trials for a wide range of diseases, which has been attributed to improvements in chemical modifications of ASO.? Incorporating a phosphorothioate (PS)-containing backbone to the ASO increased the stability and resistance of the oligonucleotides to nucleolytic degradation. ${ }^{1}$ Second-
Ferrata Storti Foundation

Haematologica 2022

Volume 107(2):519-531

\section{Correspondence:}

MARTINA LUNDBERG SLINGSBY

martina.slingsby@gmail.com

Received: June 22, 2020.

Accepted: February 2, 2021

Pre-published: February 11, 2021.

https://doi.org/10.3324/haematol.2020.260059

(C)2022 Ferrata Storti Foundation

Material published in Haematologica is covered by copyright. All rights are reserved to the Ferrata Storti Foundation. Use of published material is allowed under the following terms and conditions:

https://creativecommons.org/licenses/by-nc/4.0/legalcode. Copies of published material are allowed for personal or internal use. Sharing published material for non-commercial purposes is subject to the following conditions:

https://creativecommons.org/licenses/by-nc/4.0/legalcode, sect. 3. Reproducing and sharing published material for commercial purposes is not allowed without permission in writing from the publisher. 
generation ASO have the PS backbone and often include 2'O-methoxyethyl (MOE) modifications, which can further reduce ASO degradation and increase affinity for the target mRNA. ${ }^{1}$

While several trials with ASO drugs have shown 2'MOEcontaining ASO drugs to be well tolerated, there have been reports of adverse events including dose-dependent thrombocytopenia, with two phenotypes having been described. ${ }^{8,9}$ Phenotype 1 is a moderate dose-dependent drop in platelet counts that is reversible with cessation of drug treatment. ${ }^{8,10}$ The Ionis integrated safety database concluded that certain sequences ( 3 out of $162^{\prime} \mathrm{MOE}$ ASO) were associated with phenotype 1 , with dose-dependent moderate $(>30 \%)$ declines in platelet count without an effect on bleeding risk. ${ }^{8}$ Phenotype 1 has also been observed in monkeys treated with $40 \%$ of the evaluated $2^{\prime} \mathrm{MOE}$ ASO, in which it was manifested as a consistent, reproducible decline of platelets over 4-6 weeks, with the platelet counts decreasing moderately (by 30-50\%) and then remaining steady. ${ }^{11}$ Phenotype 2 is a sporadic severe drop in platelet count (clinical thrombocytopenia) and is often not reproducible (for the same 2'MOE ASO), but appears to be dose-dependent. ${ }^{11}$ Treatment with the 2'MOE ASO inotersen induced phenotype 2 in a few individuals who were predisposed, in relation to their underlying disease, and was shown to be dependent on platelet antibodies. ${ }^{12}$

This paper will focus on the underlying cause of the more prevalent phenotype 1 .

Drug-induced thrombocytopenia can stem from decreased production of platelets in the bone marrow and/or increased destruction and clearance of platelets from peripheral blood. ${ }^{13}$ To examine the in vivo effects on platelet counts, monkeys were treated with the 2'MOE ASO 104838 for 12 weeks. ${ }^{10}$ Platelet counts decreased $~ 50 \%$ in all monkeys by day 30 , and four out of five monkeys had decreases in platelet counts consistent with phenotype 1 . The drops in platelet counts were attributed to a $60-80 \%$ increase in platelet sequestration in liver and spleen, caused by either increased phagocytosis of platelets or trapping of platelets on the reticuloendothelial surface of these organs. ${ }^{10}$ Thrombopoietin levels were not altered in the monkeys and bone marrow megakaryocyte morphology, cell density and maturation appeared normal. These findings suggested that the mild thrombocytopenia was due to increased platelet destruction or splenic sequestration and not to diminished platelet production). ${ }^{10}$

There have been few studies investigating the direct effects of ASO on human platelets. Flierl et al. showed that incubating human platelets with an oligonucleotide PSODN 2395 (without 2'MOE modifications), led to platelet activation (increased platelet P-selectin surface expression)..$^{14}$ The same study established that the PS backbone modification of ODN 2395 is a significant driver of this drug's effects on platelets, through binding and activation of the platelet receptor glycoprotein VI (GPVI), by enhanced GPVI receptor clustering/dimerization. ${ }^{14}$ Sewing et al. supported these findings and demonstrated that ASO containing locked nucleic acid modifications had reduced binding to GPVI and platelet activation. ${ }^{15}$ These studies did not include 2'MOE-modified ASO. Considering that most ASO in clinical use and in the drug development pipeline are $2^{\prime} \mathrm{MOE} \mathrm{ASO}$, it is imperative to understand how 2'MOE modifications affect platelet responses in humans. Furthermore, it remains unknown whether ASO affect proplatelet production from megakaryocytes and to what extent 2'MOE-modified PS-ASO share the platelet-activating effects of PS-ODN 2395 on human platelets. In the current study, we therefore focused on investigating the in vitro effects of $2^{\prime} \mathrm{MOE}$ ASO on proplatelet production from human cord blood-derived megakaryocytes and studied the direct effects of 2'MOE ASO on human platelets as well as interactions with immune cells. Specifically, we tested 2 'MOE ASO 104838, which is known to cause phenotype 1 platelet count reductions. ${ }^{10,16} \mathrm{We}$ also included 2'MOE ASO 501861, with unknown effects on human platelets, and 2'MOE ASO 487660, which has not been associated with reductions in platelet counts in monkeys. CpG ASO 2395 and another CpG 2'MOE ASO 120704 were included for comparison for their known platelet-activating effects. ${ }^{10,14}$

\section{Methods}

\section{Human and mouse megakaryocyte cultures and proplatelet analysis}

Human cord blood-derived primary $\mathrm{CD} 34^{+}$cells were cultured and mature megakaryocytes were purified by magnetic bead separation on day 11 as previously described. ${ }^{17}$ Mouse megakaryocytes were derived from fetal liver cultures extracted from CD-1 pregnant mice at day 13.5 of gestation. ${ }^{18}$ Human-derived, or mouse-derived megakaryocytes were plated on a 96-well half-area plate (Greiner Bio one 675101), followed by addition of ASO (5 $\mu \mathrm{M}$ ) and imaged at hourly intervals for $24 \mathrm{~h}$ using an IncuCyte Live Cell Analysis System (IncuCyte Zoom). These images were analyzed for the percentage of megakaryocytes producing proplatelets, as well as the area containing proplatelets using Ilastik (version 1.3.0) and Cell Profiler (version 3.0.0) as described previously. ${ }^{19}$

\section{Blood collection}

Blood was collected from healthy male and female human donors, after informed consent and institutional review board approval (2012P001526), in accordance with the Declaration of Helsinki. Washed platelets, platelet-rich plasma or whole blood was treated for $30 \mathrm{~min}$ (platelet studies) or $6 \mathrm{~h}$ (cytokine release experiments) with 1, 5 or $10 \mu \mathrm{M}$ ASO. Previous studies have shown that ASO-induced activation of platelets is concentrationdependent. ${ }^{10,14,15} \mathrm{~A}$ therapeutically relevant dose of ASO, i.e., 300 $\mathrm{mg}$, administered subcutaneously results in a Cmax (maximum concentration that a drug achieves after dosing) of $1-2 \mu \mathrm{M}^{20}$

\section{Antisense oligonucleotides}

ASO were synthesized at Ionis Pharmaceuticals, Inc. (Carlsbad, CA, USA), purified by reverse-phase high-performance liquid chromatography and formulated in $10 \mu \mathrm{M}$ HEPES. Five ASO (all with a PS backbone) were included: three with 2'-MOE sequences; 487660 (no effects on platelet count), 104838 (associated with phenotype 1),, 10501861 (effects unknown) and two with CpG sequences; 120704 and ODN 2395 (known to activate platelets) ${ }^{10,14}$ (Table 1). More details of the Methods can be found in the Online Supplementary Material.

\section{Results}

Proplatelet formation from human cord blood or murine fetal liver-derived megakaryocytes is not inhibited by 2'MOE or CpG ASO treatment

To investigate whether the ASO induced thrombocy- 
Table 1. Antisense oligonucleotides included in this study.

\begin{tabular}{|c|c|c|c|c|}
\hline $\begin{array}{l}\text { lonis } \\
\text { ASO }\end{array}$ & Back bone & Length & PS load* & Platelet side effects \\
\hline 487660 & 2'MOE PS & 20 & 19 & $\begin{array}{c}\text { No reported drops } \\
\text { in platelet count }\end{array}$ \\
\hline 487660 & \multirow{2}{*}{\multicolumn{4}{|c|}{ 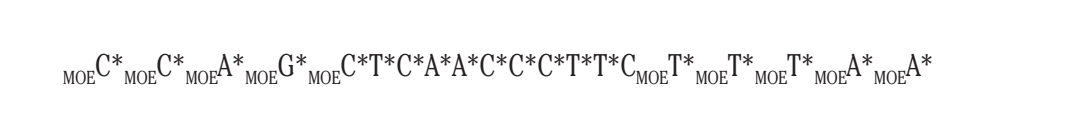 }} \\
\hline $\begin{array}{l}\text { Sequence } \\
\left(53^{\prime}\right)\end{array}$ & & & & \\
\hline 104838 & 2'MOE PS & 20 & 19 & $\begin{array}{c}\text { Phenotype 1-moderate } \\
\text { drops in platelet } \\
\text { count }^{8,10}\end{array}$ \\
\hline \multicolumn{5}{|l|}{104838} \\
\hline $\begin{array}{l}\text { Sequence } \\
\left(5^{\prime} 3^{\prime}\right)\end{array}$ & \multicolumn{4}{|c|}{ 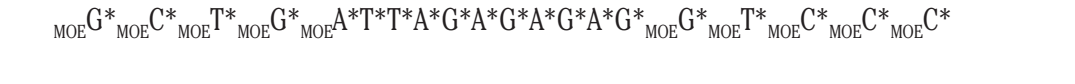 } \\
\hline
\end{tabular}

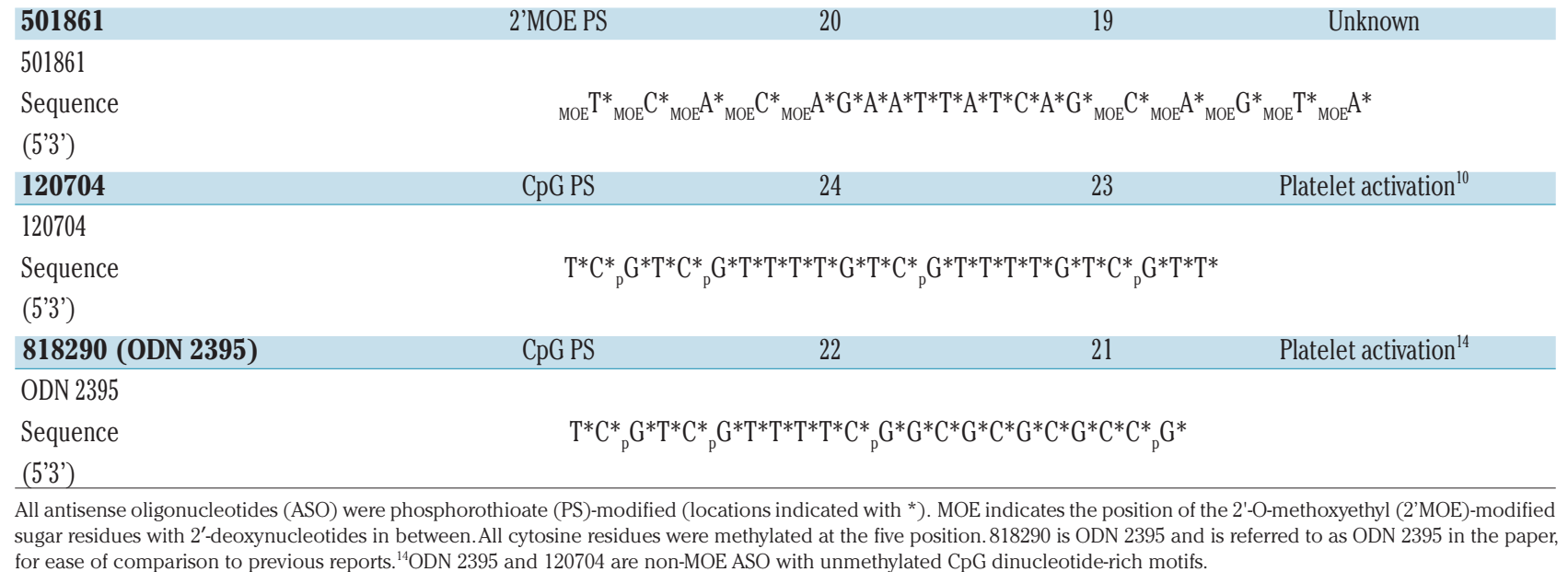

topenia by affecting proplatelet production from megakaryocytes, human cord blood-derived mature megakaryocytes were incubated with $5 \mu \mathrm{M}$ ASO for $24 \mathrm{~h}$. Puromycin, an inhibitor of protein synthesis known to impair proplatelet production, ${ }^{21,22}$ had an inhibitory effect on both human- and murine-derived megakaryocytes (Figure 1A, B). Compared to the vehicle, none of the ASO tested lowered proplatelet counts from the human-derived megakaryocytes (Figure 1A). Similarly, there was no decrease in percent proplatelet producing murine fetal liverderived megakaryocytes following incubation with any of the ASO (Figure 1B). The CpG ASO 120704 slightly increased proplatelet counts in the human-derived megakaryocytes after $22 \mathrm{~h}(117 \pm 5 v s$. vehicle $79 \pm 7)$ (Figure $1 \mathrm{~A})$. There was also a small increase in proplatelet-producing murine megakaryocytes after $24 \mathrm{~h}$ (Figure 1B); vehicle

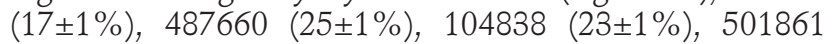
$(25 \pm 2 \%), 120704(23 \pm 1 \%)$, and ODN 2395 (26 $\pm 1 \%)$. Representative images of proplatelet production at $0,8,16$ and $24 \mathrm{~h}$ by murine megakaryocytes treated with 2 'MOE ASO 104838 or CpG ASO ODN 2395 appeared comparable to those of megakaryocytes treated with the vehicle (Figure 1C).

\section{Human platelets internalize both 2'MOE and CpG ASO}

Given that the ASO did not appear to inhibit proplatelet production, we focused on examining the direct effects of ASO on human platelets. We used immune-electron microscopy to visualize how ASO (at $5 \mu \mathrm{M}$ ) interact with human washed platelets. ASO with a PS backbone are hydrophilic, poly-anionic molecules with a high degree of plasma protein binding (typically $>90 \%$ ), and circulate transiently in the blood before interacting with cell-surface proteins and typically gaining entry into cells by endocytosis. ${ }^{23}$ The plasma-free conditions in the experiments shown in Figure 2 were intended to maximize visualization of ASO binding to platelets. Electron micrographs revealed that ASO immunogold staining was either localized to the platelet plasma membrane or, when internalized, to the cytoplasm with sporadic staining of internal membranes and granules (Figure 2B-F). The 2'MOE ASO 487660 appeared to stain less than the other ASO, especially at the plasma membrane (Figure 2B-F).

To exclude any direct platelet cytotoxicity of $\mathrm{ASO}$, we performed a lactate dehydrogenase leakage assay in washed platelets and confirmed that none of the ASO was cytotoxic at doses of 1 or $5 \mu \mathrm{M}$ (Online Supplementary Figure S1).

\section{2'MOE ASO (104838 and 501861) and CpG ASO increase platelet P-selectin expression in platelet-rich plasma and whole blood}

To establish whether the ASO activate human platelets directly, we investigated the effects on platelet surface Pselectin levels using flow cytometry (Figure 3). Plateletrich plasma treated with the positive control thrombin receptor activating peptide (TRAP) showed a significant increase in P-selectin surface expression, while the $2^{\prime} \mathrm{MOE}$ ASO 487660 (which does not affect platelet count) did not increase platelet P-selectin compared to vehicle (Figure $3 \mathrm{~A})$. Platelet activation nearly doubled after treatment 
A

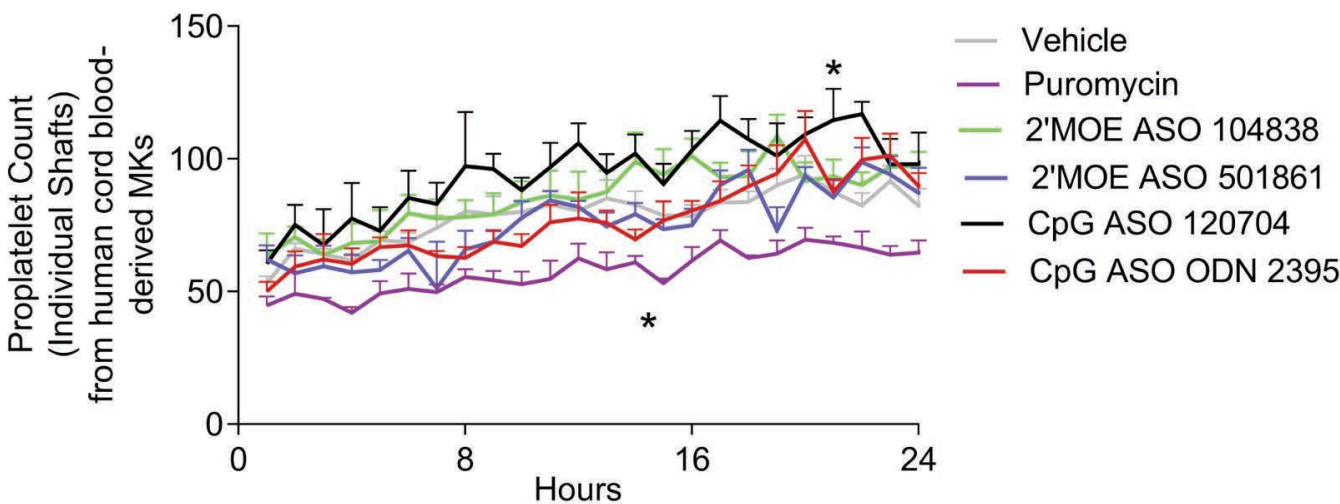

B

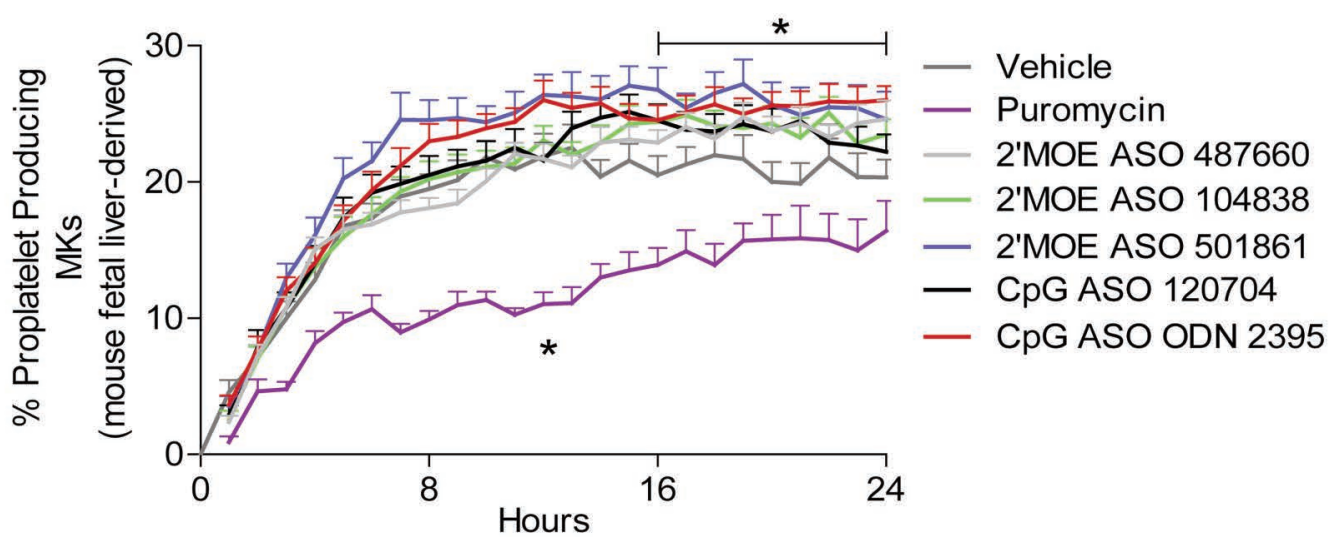

C

0 Hours

8 Hours

16 Hours
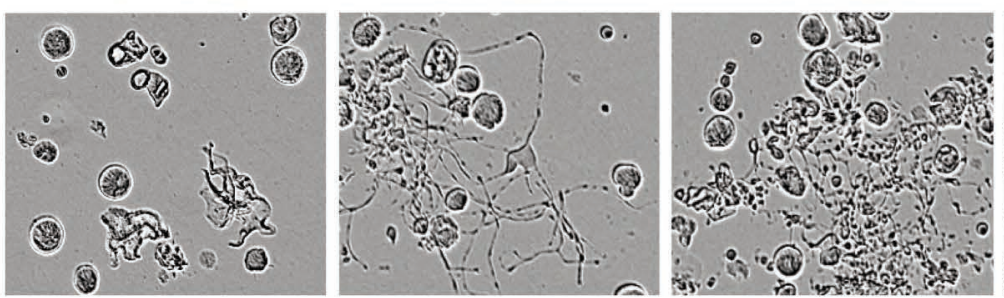

24 Hours

Vehicle
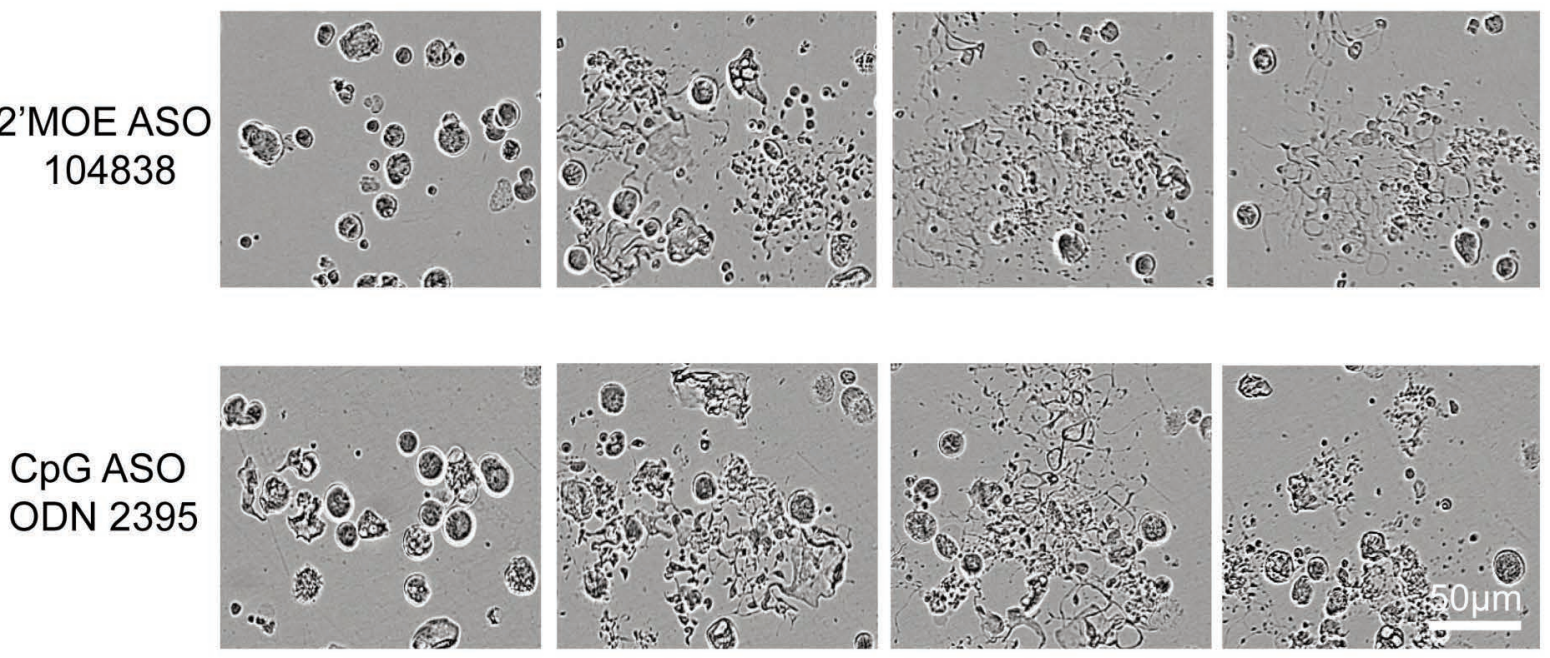

Figure 1. The effect of antisense oligonucleotides on proplatelet production by megakaryocytes derived from human cord blood and mouse fetal liver. Human and mouse megakaryocytes (MK) were treated with vehicle (HEPES, $10 \mathrm{mM}$ ), puromycin (100 nM, a known inhibitor of proplatelet production), or $5 \mu \mathrm{M}$ of the 2'MOE antisense oligonucleotides (ASO) 104838 or 501861 or the CpG ASO 120704 or ODN 2395 for 24 h using the IncuCyte Live Cell Analysis System and analyzed with Ilastik and Cell Profiler. (A) Proplatelet count: individual count of proplatelet protrusions that emanate from the human cord blood-derived MK body, over the course of the 24 $\mathrm{h}$ incubation. (B) Percent of proplatelet-producing mouse fetal liver-derived MK treated with the above treatments as well as the $2{ }^{\prime} \mathrm{MOE}$ ASO 487660 . * $P<0.05$ by twoway analysis of variance with Bonferroni post-test ( $n=4$ repeat runs). (C) Representative brightfield images at 20x magnification, of proplatelet-producing mouse fetal liver-derived MK at $0 \mathrm{~h}, 8 \mathrm{~h}, 16 \mathrm{~h}$ and $24 \mathrm{~h}$ treated with vehicle (HEPES), the 2'MOE ASO 104838 or the CpG ASO ODN 2395 . Scale bars indicate 50 um. 
with $5 \mu \mathrm{M}$ of the 2'MOE ASO 104838 and 501861 $(19 \pm 3 \%$ and $20 \pm 3 \%$, respectively), compared to vehicle $(10 \pm 2 \%)$; the CpG ASO 120704 and ODN 2395 activated the platelets more potently $(38 \pm 3 \%$ and $38 \pm 4 \%$, respectively) (Figure 3A).

We also studied platelet activation in whole blood, in which platelet $\mathrm{P}$-selectin was elevated by the platelet agonists TRAP and collagen (Figure $3 \mathrm{~B}$ ). Pre-treatment with a Spleen tyrosine kinase (SYK) inhibitor (PRT-060318) decreased collagen, but not TRAP activation since SYK is downstream of the collagen receptor GPVI signaling in platelets (Figure $3 \mathrm{~B}$ ). The selective inhibitory effect on collagen signaling is demonstrated more clearly in Figure 6A. The 2'MOE ASO 104838 and 501861 had mild plateletactivating effects in whole blood (similar to that of collagen) whereas the CpG ASO 120704 and ODN 2395 had stronger effects (comparable to the effect of TRAP) (Figure 3B). Pre-treatment with the SYK inhibitor blocked the ASO-induced P-selectin expression (Figure $3 \mathrm{~B}$ ).

\section{Responsiveness to ASO treatment is strongly correlated to individual GPVI levels}

Since we noticed donor-to-donor variability in the responsiveness to ASO treatment (Figure 3B), we investigated whether this could be related to differential platelet surface expression of GPVI receptors, which we measured by flow cytometry. Basal platelet GPVI levels varied between donors and activating the platelet-rich plasma with TRAP reduced the platelet GPVI levels (Online Supplementary Figure S2), consistent with reports of GPVI shedding upon platelet activation. ${ }^{24}$ Pearson correlation analysis showed a strong positive correlation (correlation
A Vehicle

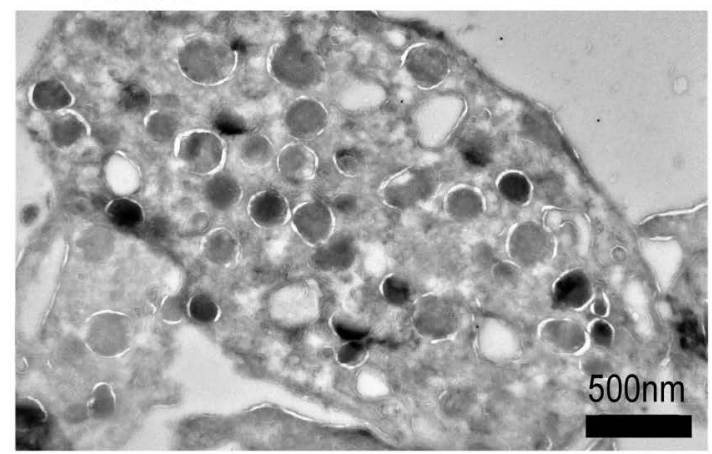

C 2'MOE ASO 104838

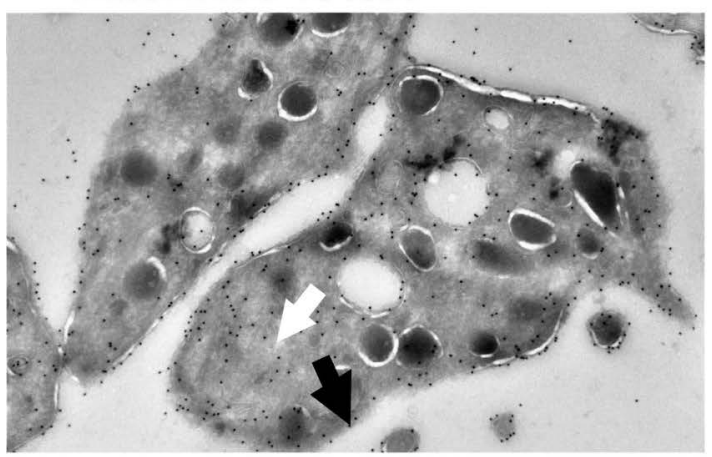

E CpG ASO 120704

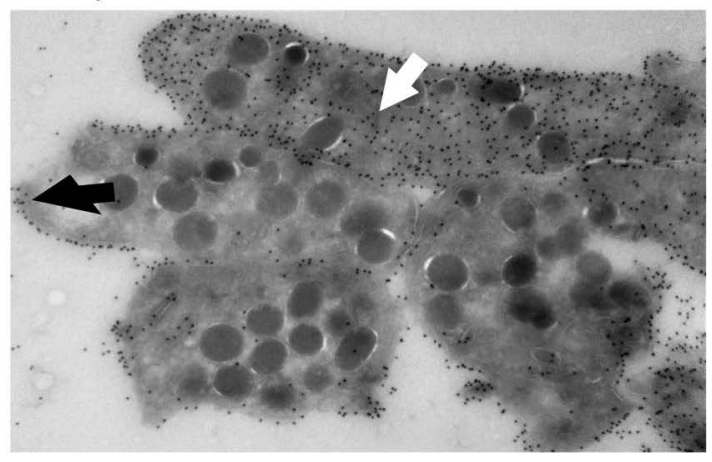

\section{B 2'MOE ASO 487660}

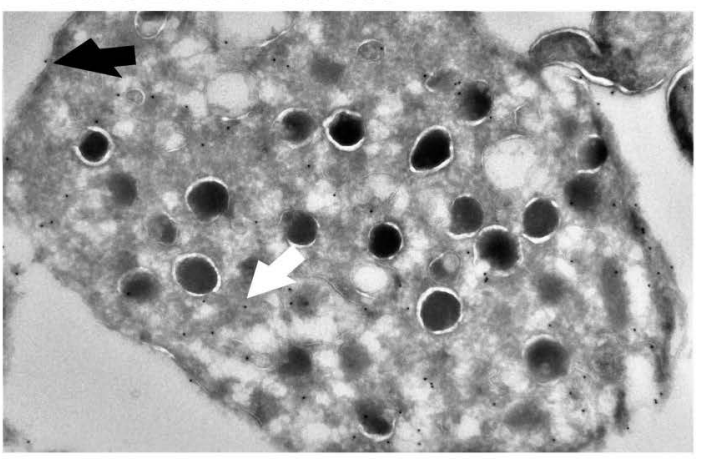

\section{2'MOE ASO 501861}

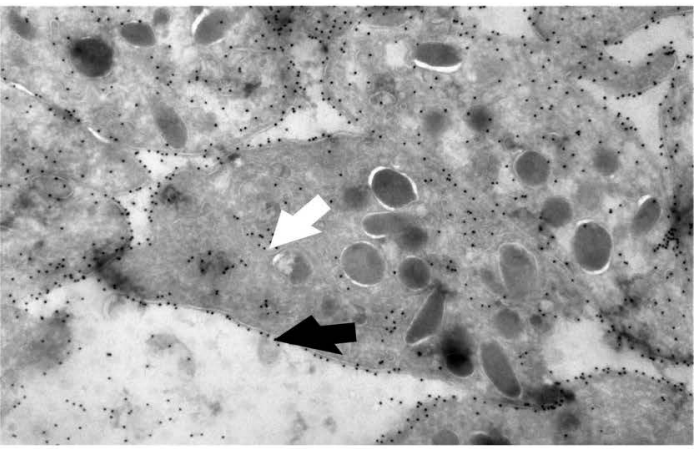

\section{F CpG ASO ODN 2395}

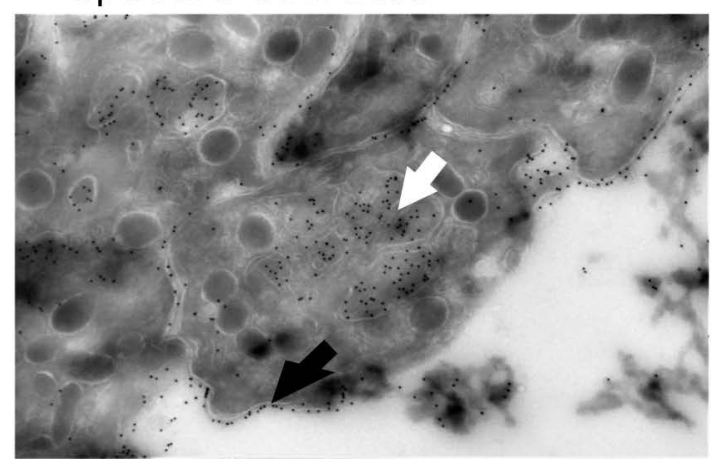

Figure 2. Uptake and localization of antisense oligonucleotides in human washed platelets. Washed platelets were incubated with a therapeutically relevant concentration of the antisense oligonucleotides (ASO) ( $5 \mu \mathrm{M})$, then fixed and stained with an anti-ASO antibody and labeled with protein A-gold. Representative electron microscopy images at 15000x of ASO localization, using anti-ASO immunogold labeling (shown as black dots) of human washed platelets treated for 30 min with (A) vehicle (HEPES, 10 mM), (B) 2'MOE ASO 487660, (C) 2'MOE ASO 104838, (D) 2'MOE ASO 501861, (E) CpG ASO 120704, or (F) CpG ASO ODN 2395 (all at 5 M). Black arrows indicate ASO localizing on the platelet membrane and white arrows indicate internalized ASO. 
A

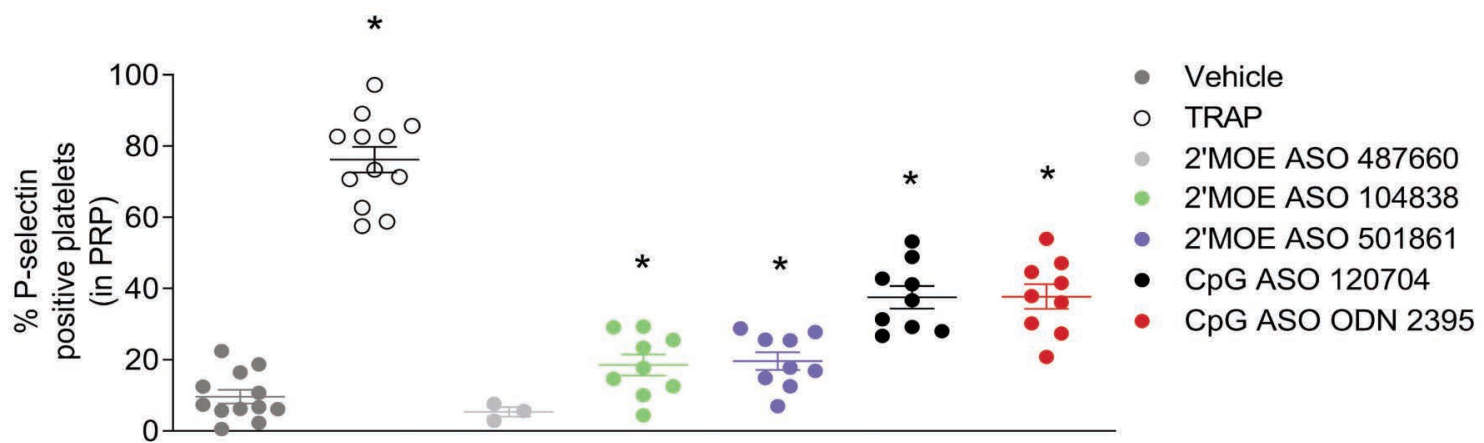

B
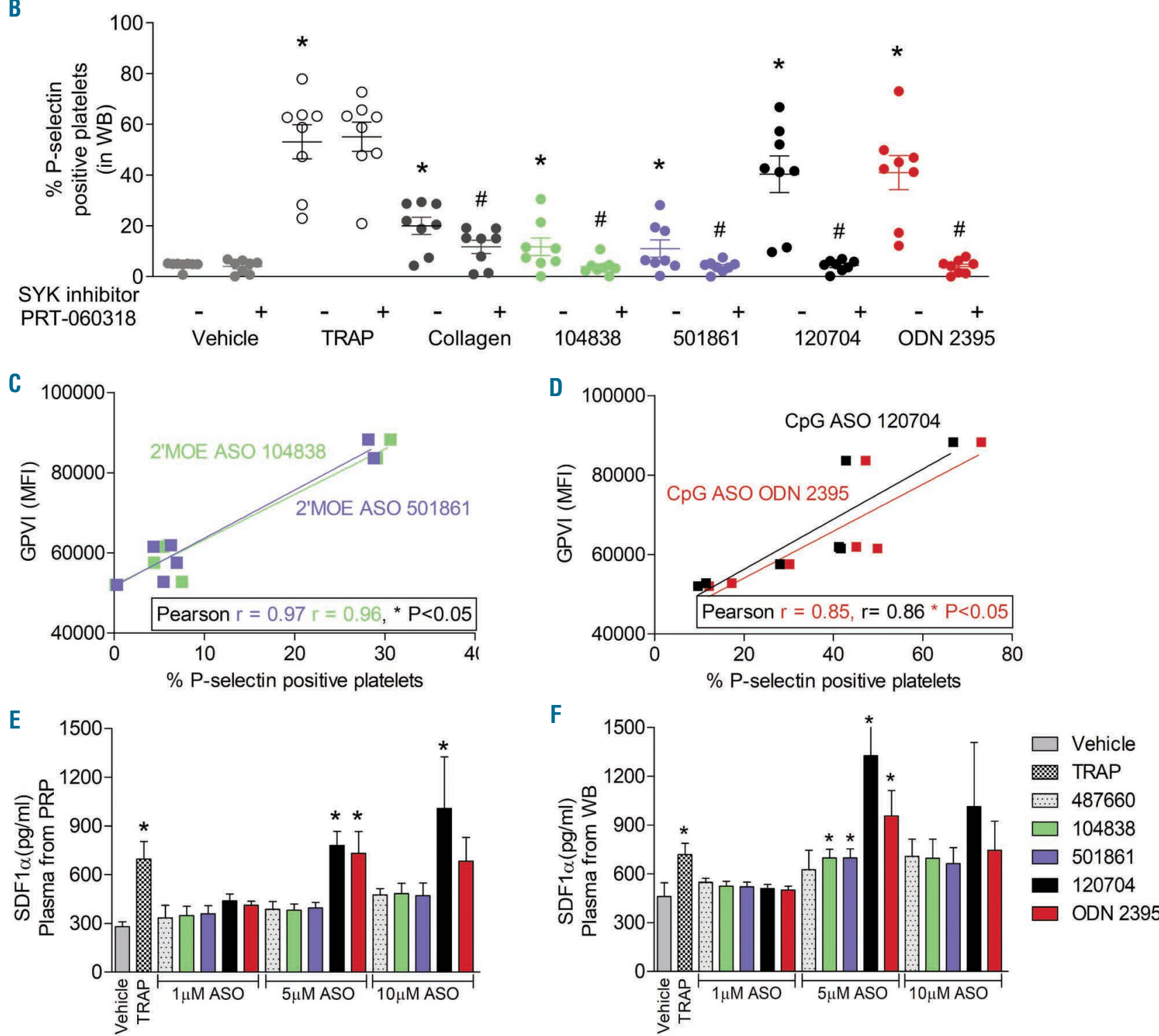

Figure 3. Effect of antisense oligonucleotides on platelet activation marker P-selectin and stromal cell derived factor $1 \alpha$ release in human platelet-rich plasma and whole blood and correlation with individual platelet glycoprotein VI receptor levels. Platelet activation was identified through increases in P-selectin expression on the platelet surface after 30 min treatment with vehicle (HEPES, $10 \mathrm{mM}$ ) or thrombin receptor activating peptide (TRAP, $25 \mu \mathrm{M}$, to activate the platelets) or $5 \mu \mathrm{M}$ of the antisense oligonucleotides (ASO): 104838 (2'MOE ASO), 501861 (2'MOE ASO), 120704 (CpG ASO) and ODN 2395 (CpG ASO), assessed by flow cytometry in (A) platelet-rich plasma (PRP) (from 9 human donors, the 2'MOE ASO 487660 was included in 3 of the experiments) and (B) whole blood (WB) (from 8 human donors) pretreated or not with the spleen tyrosine kinase (SYK) inhibitor PRT-060318 (10 $\mu \mathrm{M})$ before addition of vehicle, TRAP $(25 \mu \mathrm{M})$ or ASO $(5 \mu \mathrm{M})$ that increased P-selectin in PRP (i.e., 104838, 501861, 120704 and ODN 2395). * $P<0.05$ compared to vehicle by one-way analysis of variance (ANOVA), with the Dunnett post-test. ${ }^{*} P<0.05$ paired Student t-test for the effect of the SYK inhibitor. (C, D) Individual donor platelet glycoprotein (GP)VI receptor levels (median fluorescence intensity, MFI) were correlated to the same individual platelet P-selectin levels after treatment with: (C) 2'MOE ASO 104838 or 501861, and (D) CpG ASO 120704 or ODN 2395, (7 human donors). $P<0.05$ by Pearson correlation analysis. (E, F) Stromal cell derived factor $1 \alpha$ (SDF1 $\alpha$ ), released from platelets upon activation, was measured by Mesoscale U-plex multiplex assay in (E) PRP and (F) WB blood treated for 30 min with vehicle (HEPES), TRAP (25 $\mu \mathrm{M})$ or 1,5 or $10 \mu \mathrm{M}$ of the ASO in blood from four human donors. ${ }^{*} P<0.05$ compared to vehicle by one-way ANOVA, with Dunnett post-test. 
coefficients: $104838 \mathrm{r}=0.97,501861 \mathrm{r}=0.96,120704 \mathrm{r}=0.85$, ODN $2395 \mathrm{r}=0.86$ ) between donors' platelet GPVI levels and their platelet activation in response ASO treatment, with the highest ASO responders also having the highest platelet GPVI levels (Figure 3 C, D).

\section{2'MOE ASO (104838 and 501861) and CpG ASO} trigger SDF1 $\alpha$ release from whole blood

As an additional marker of $\alpha$-granule release from acti- vated platelets, we measured secreted plasma levels of stromal cell derived factor $1 \alpha$ (SDF1 $\alpha$ ) in ASO-treated platelet-rich plasma and whole blood. TRAP stimulated SDF1 $\alpha$ release in both platelet-rich plasma and whole blood (Figure 3 E, F). Consistent with the pattern of the Pselectin effects, the CpG ASO 120704 and ODN 2395 triggered robust release of SDF1 $\alpha$ in platelet-rich plasma and whole blood in a concentration-dependent manner: thus the effect could be seen with doses of 5 and $10 \mu \mathrm{M}$, but
A

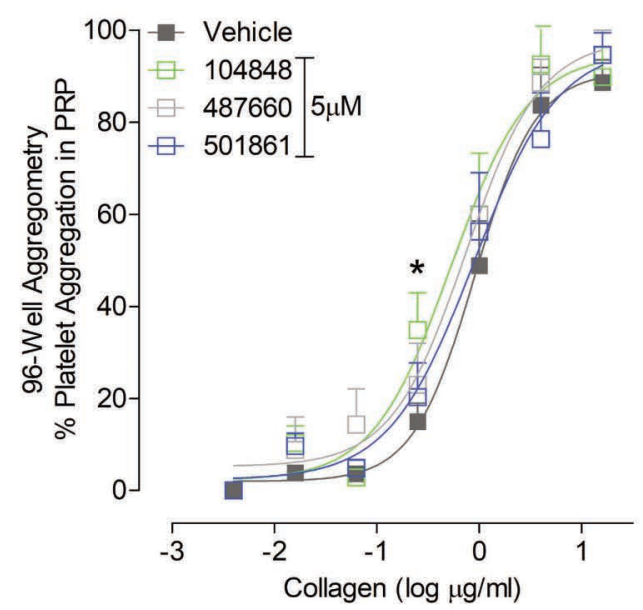

C

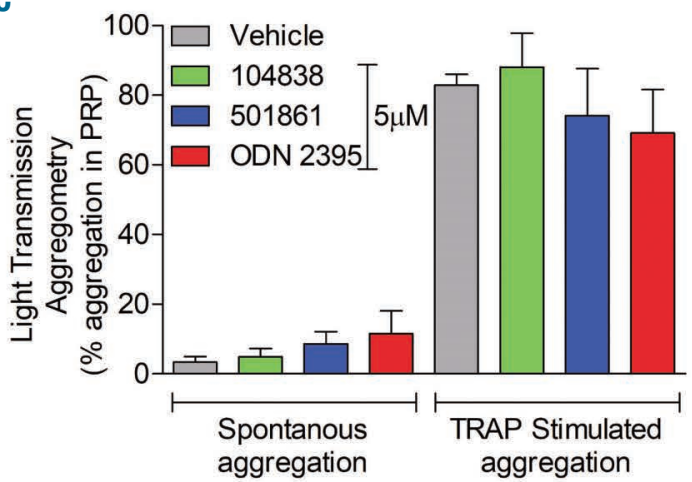

$E$

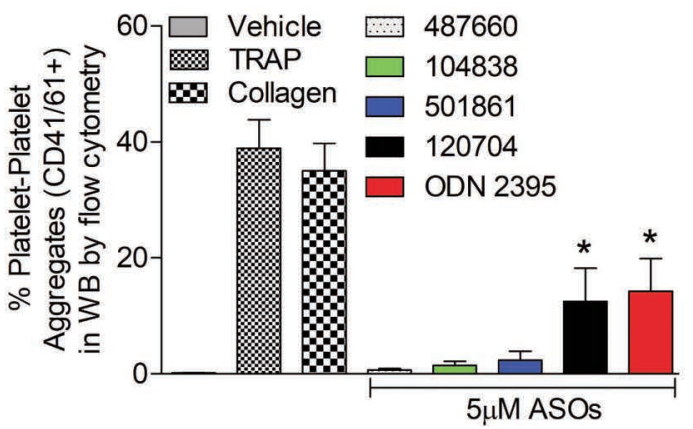

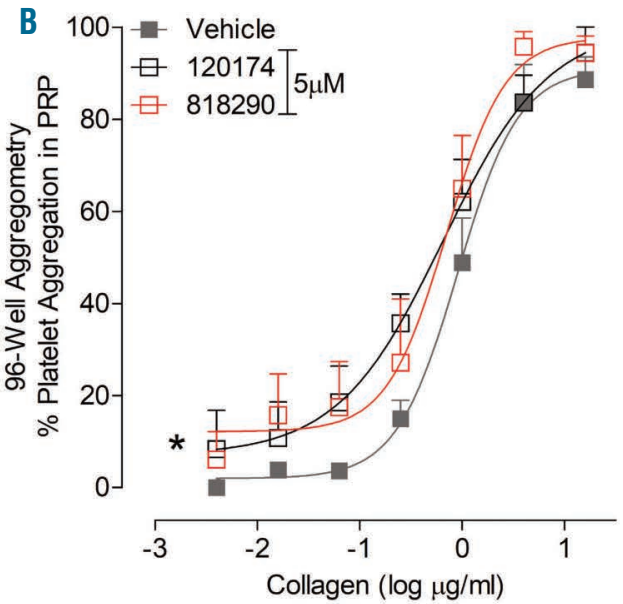

D

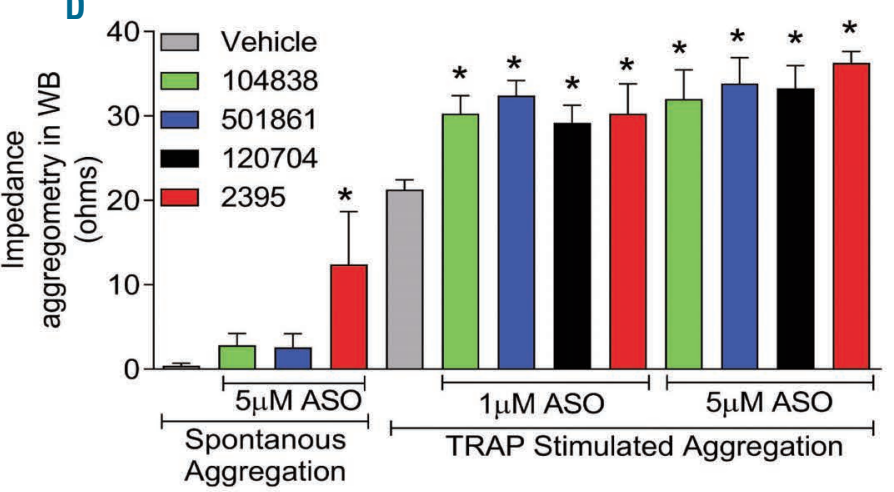

$\mathbf{F}$

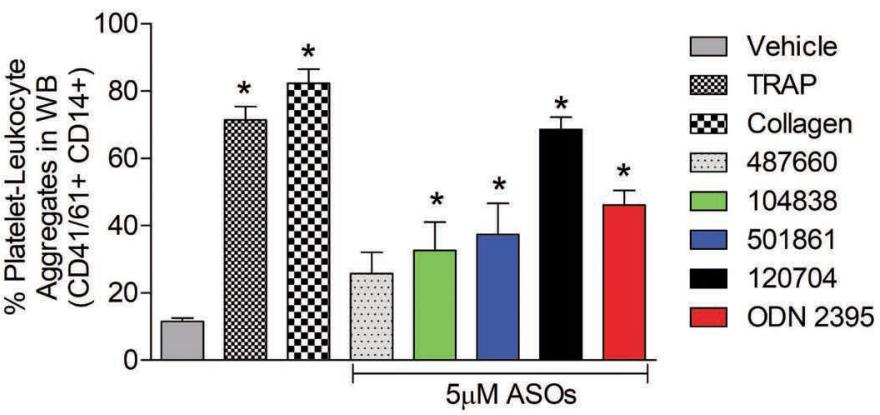

Figure 4. Platelet aggregation in human platelet-rich plasma and whole blood treated with antisense oligonucleotides. (A, B) Ninety-six-well platelet aggregometry was used to generate full concentration-response curves to the platelet agonist collagen after incubating platelet-rich plasma (PRP) with vehicle or (A) $5 \mu \mathrm{M}$ of the 2'MOE antisense oligonucleotides (ASO) 487660, 104838 and 501861 or (B) the CpG ASO 120704 or ODN 2395. *P<0.05 by two-way analysis of variance (ANOVA) with Bonferroni post-test, compared to vehicle (HEPES, $10 \mathrm{mM}$ ), $n=6-8$ human donors. (C) Platelet aggregation was also assessed using traditional light transmission aggregometry (LTA) by incubating PRP ( $n=8$ human donors) with the ASO for 30 min at 1200 rpm stirring speed without stimulation (to detect spontaneous aggregation) or after stimulation with the platelet agonist thrombin receptor activating peptide (TRAP, $25 \mu \mathrm{M}$ ). (D) Impedance aggregometry was used to analyze platelet aggregation by incubating whole blood (WB) ( $n=7$ human donors) with the ASO (at 1 and $5 \mu \mathrm{M}$ ) for 30 min at 1200 rpm stirring speed, without stimulation, or TRAP $(25 \mu \mathrm{M})$ stimulated aggregation. (E) Platelet-platelet aggregates in WB ( $\mathrm{n}=7$ human donors) treated with vehicle (HEPES, $10 \mathrm{mM})$, collagen $(20 \mu \mathrm{g} / \mathrm{mL})$, TRAP $(25 \mu \mathrm{M})$ or ASO $(5 \mu \mathrm{M})$ were analyzed using flow cytometry. (F) Platelet-leukocyte aggregates (platelet marker CD41/61 $1^{+}$leukocyte marker CD14 ${ }^{+}$) were analyzed in WB ( $n=6$ human donors) treated with vehicle (HEPES, $10 \mathrm{mM})$, collagen $(20 \mu \mathrm{g} / \mathrm{mL})$, TRAP $(25 \mu \mathrm{M})$, or ASO $(5 \mu \mathrm{M})$, using flow cytometry. *P<0.05 compared to vehicle by one-way ANOVA, Dunnett post-test (C, D, E, F). 

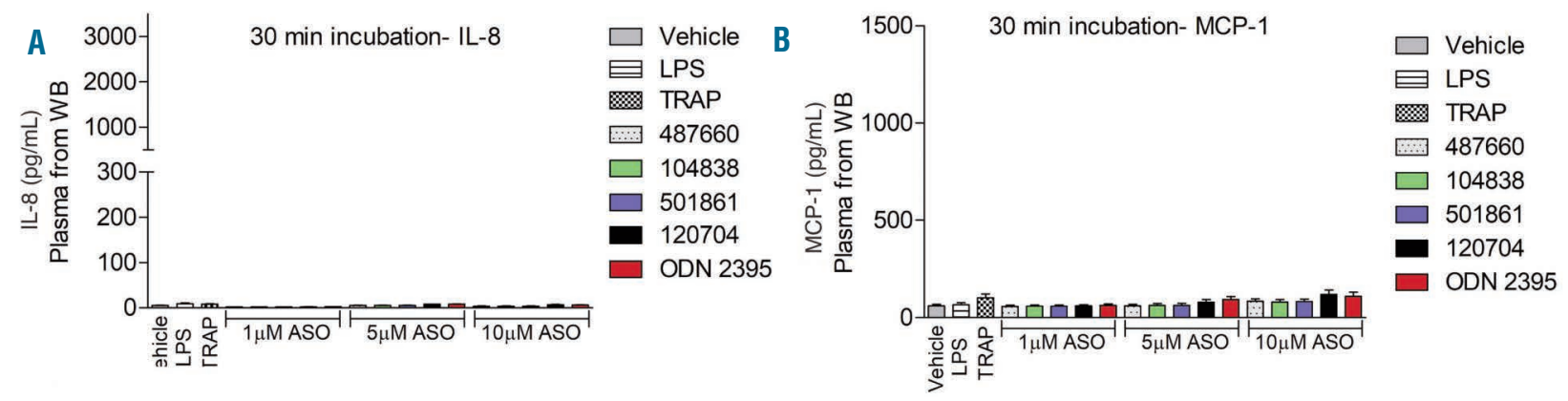

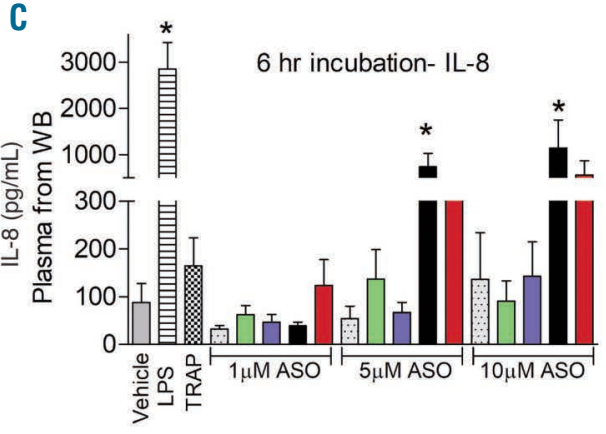

$\mathbf{E}$

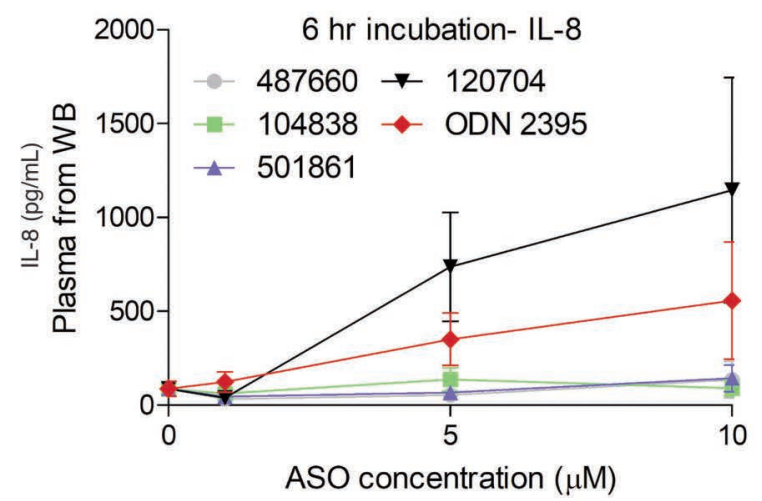

D

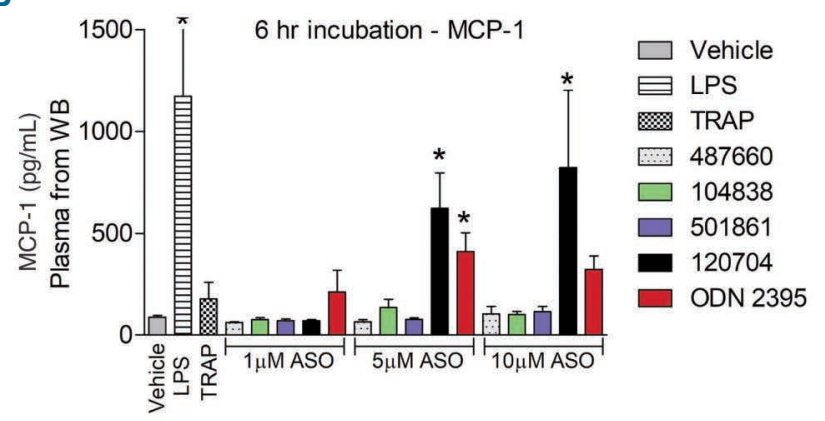

$\mathbf{F}$

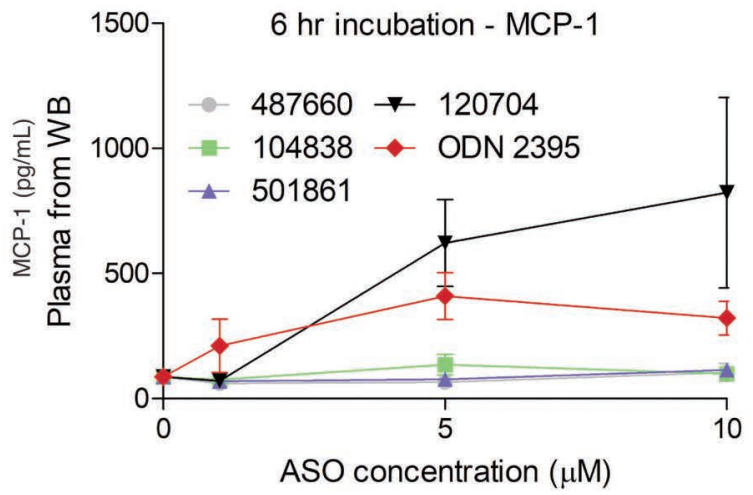

Figure 5. Immunostimulatory effects after 30 minutes and 6 hours in antisense oligonucleotide-treated whole blood. Whole blood (WB) from four human donors was incubated with vehicle (HEPES, $10 \mathrm{mM}$ ), thrombin receptor activating peptide (TRAP, $25 \mu \mathrm{M}$ ), lipopolysaccharide (LPS $0.01 \mathrm{mg} / \mathrm{mL}$ ) or $1,5 \mathrm{or} 10 \mu \mathrm{M}$ of the antisense oligonucleotides (ASO). Interleukin 8 (IL-8) and monocyte chemotactic protein-1 (MCP-1) release was measured in plasma (pg/mL), using an MSD U-plex assay, after incubation for $30 \mathrm{~min}$ and $6 \mathrm{~h}$ at $37^{\circ} \mathrm{C}$ in $5 \% \mathrm{CO}_{2}$. (A) IL-8 after $30 \mathrm{~min}$. (B) MCP-1 after $30 \mathrm{~min}$. (C) IL-8 after $6 \mathrm{~h}$. (D) MCP-1 after $6 \mathrm{~h}$. *P<0.05 compared to vehicle by one-way analysis of variance, Dunnett post-test. Concentration-response curves for (E) IL- 8 release after $6 \mathrm{~h}$ and (F) MCP-1 release after $6 \mathrm{~h}$.

not at a dose of $1 \mu \mathrm{M}$ (Figure $3 \mathrm{E}, \mathrm{F}$ ). The 2 ' $\mathrm{MOE}$ ASO 104838 and 501861 triggered a small release at $5 \mu \mathrm{M}$ in whole blood only (Figure 3F). The 2'MOE ASO 487660 did not trigger SDF1 $\alpha$ release at any concentration tested, in either platelet-rich plasma or whole blood (Figure 3E, F).

\section{2'MOE ASO 104838 increases platelet reactivity to collagen}

To investigate whether the platelet activation documented in Figure 3 would translate into an effect on platelet aggregation, we performed 96-well aggregometry and traditional light transmission aggregometry under both unstimulated and stimulated conditions (Figure 4AD). The 2'MOE ASO 104838 triggered a small potentiating effect on collagen-induced platelet aggregation, but this effect was not seen after treatment with the other two 2'MOE ASO (Figure 4A). The CpG ASO 120704 and ODN 2395 also increased platelet reactivity to collagen (Figure $4 \mathrm{~B})$, including $\sim 40 \%$ spontaneous aggregation in two donors with high platelet GPVI levels (Online Supplementary Figure S2). However, no significant spontaneous aggregation was detected by light transmission aggregometry in any of the ASO-treated platelet-rich plasma samples and stimulating the $5 \mu \mathrm{M}$ ASO-treated samples with TRAP resulted in a similar light transmission aggregometry-measured aggregation response to that following stimulation with the vehicle (Figure 4C). Differences in the mechanical shear stress environment in 96-well aggregometry versus light transmission aggregometry ${ }^{25}$ may explain the discrepancy in results between these assays.

2'MOE ASO (104838 and 501861) and CpG ASO potentiate platelet reactivity to TRAP in whole

blood impedance aggregometry

When whole blood was incubated with $5 \mu \mathrm{M}$ of the CpG ASO ODN 2395 in an impedance aggregometer, spontaneous aggregation was detected over the course of 
A

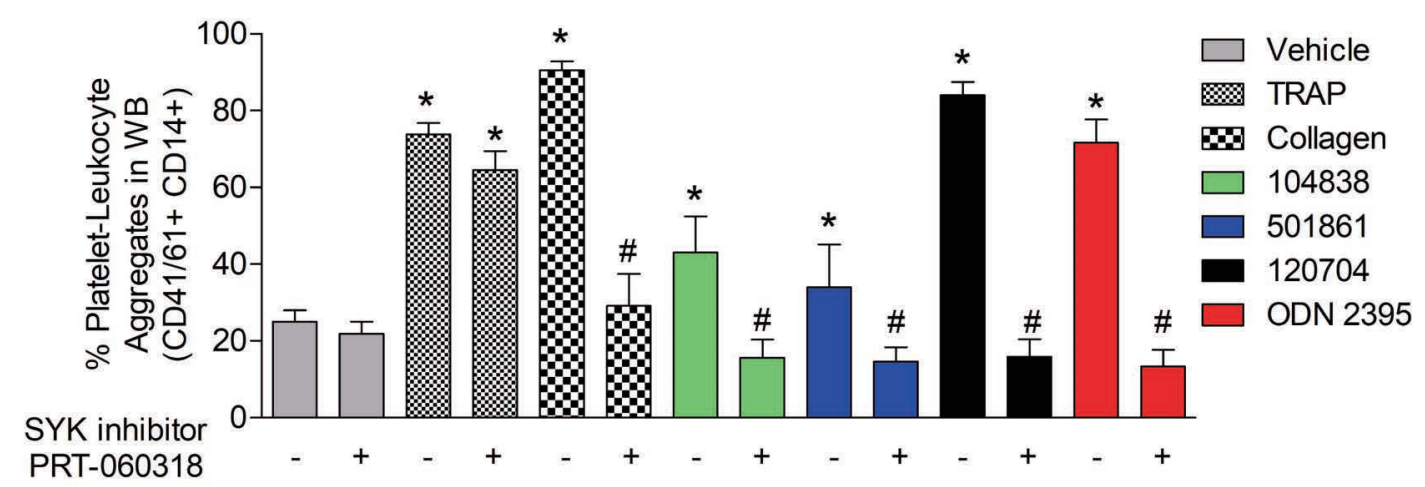

B
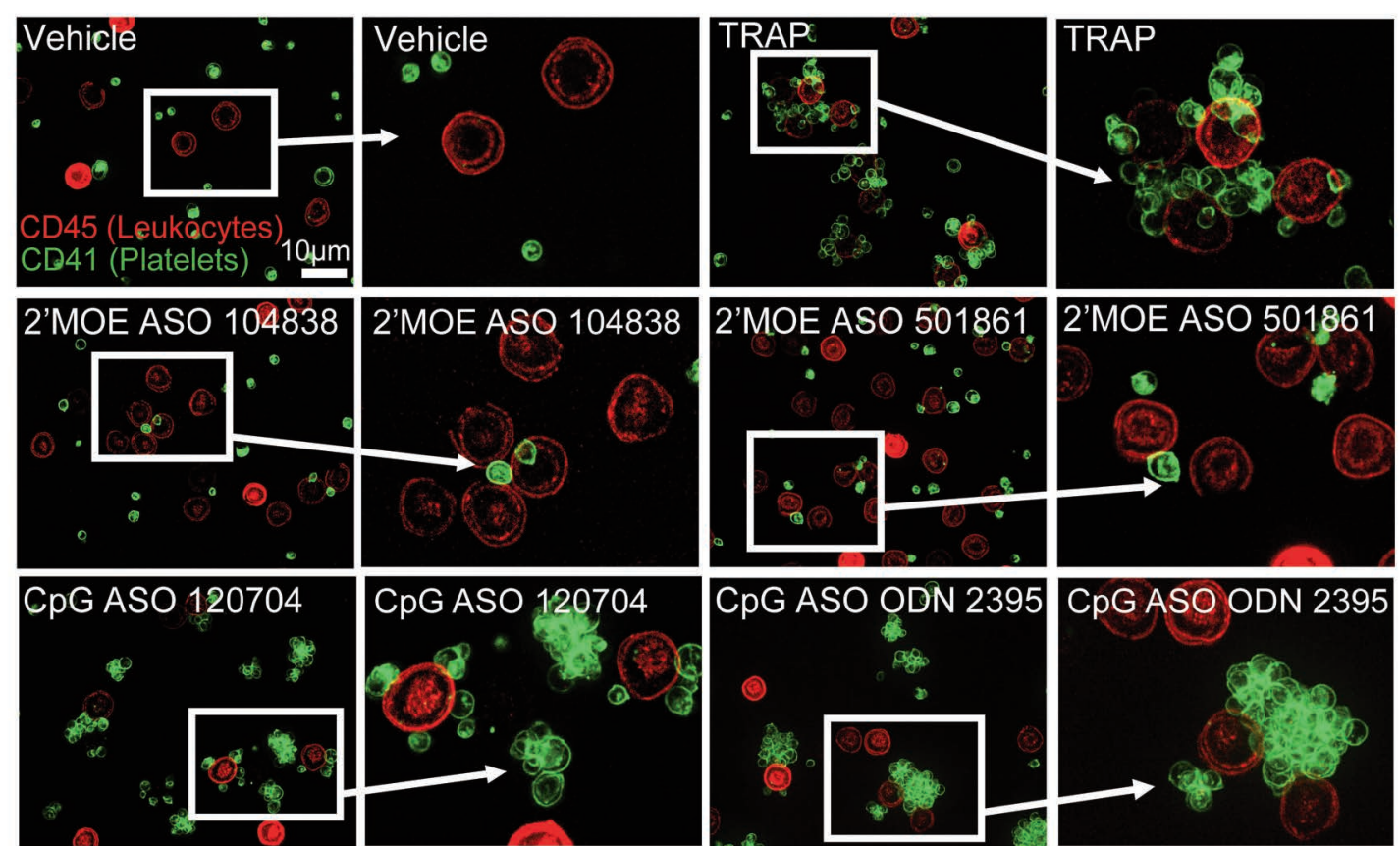

Figure 6. SYK inhibition of the formation of platelet-leukocyte aggregates in whole blood treated with antisense oligonucleotides. Whole blood (WB) from four human donors was pretreated or not with a spleen tyrosine kinase (SYK) inhibitor (PRT-060318, $10 \mu \mathrm{M})$ followed by vehicle (10 mM HEPES), thrombin receptor activating peptide (TRAP, $25 \mu \mathrm{M})$, collagen (20 $\mathrm{\mu g} / \mathrm{mL}$ ), or the 2'MOE non CpG antisense oligonucleotides (ASO): 104838 or 501861, or the CpG ASO: 120704 or ODN 2395 (all at $5 \mu \mathrm{M}$ ) and analyzed by flow cytometry for: (A) platelet-leukocyte aggregates (PLA, platelet marker CD41/61 $1^{+}$, leukocyte marker $C D 14^{+}$). $* P<0.05$ compared to vehicle by one-way analysis of variance (ANOVA), Dunnett post-test, ${ }^{\#} P<0.05$ paired Student $t$-test for the effect of the SYK inhibitor. (B) Representative confocal images of platelet-leukocyte aggregates in fixed and fluorescently labeled WB. Red represents CD45 (leukocyte marker), green represents CD41/61 (platelet marker). The white boxes indicate the location of the zoomed-in part of image shown to the right. Scale bar $=10 \mu \mathrm{m}$.

the 30 min incubation $(12 \pm 6 \Omega v s .0 .4 \pm 0.3 \Omega$ with vehicle) (Figure 4D). When platelet-platelet interactions were studied in ASO-treated (but otherwise unstimulated) whole blood samples using flow cytometry, only the CpG ASO 120704 and ODN 2395 had significantly more plateletplatelet aggregates $(13 \pm 6$ and $14 \pm 6 \%$, respectively, vs. $0.2 \pm 0.04 \%$ with vehicle) (Figure $4 \mathrm{E}$ ), consistent with the impedance aggregometry results. After stimulation with TRAP, whole blood aggregation was potentiated to a similar level in both the 2'MOE ASO (104838 and 501861) and the CpG (120704 and ODN 2395)-treated samples at both 1 and $5 \mu \mathrm{M}$ (Figure 4D).

\section{2'MOE ASO (104838 and 501861) and CpG ASO}

trigger formation of platelet-leukocyte aggregates in unstimulated whole blood

The impedance aggregometry results led us to hypothesize that the enhancement of whole blood aggregation was perhaps not solely driven by homogenous plateletplatelet aggregates but could also contain heterogeneous platelet-leukocyte aggregates. ${ }^{26} \mathrm{We}$, therefore, analyzed ASO-treated (but otherwise unstimulated) whole blood for the formation of platelet-leukocyte aggregates by flow cytometry (Figure 4F). TRAP and collagen triggered substantial formation of platelet-leukocyte aggregates, whereas the 2'MOE ASO 487660 evoked a similar response to that produced by the vehicle (Figure 4F). However, whole blood treated with 2'MOE ASO 104838 and 501861 and the CpG 120704 and ODN 2395 had more platelet-leukocyte aggregates $(33 \pm 8,37 \pm 9,69 \pm 4$, and $46 \pm 4 \%$, respectively) than vehicle-treated whole blood (12 $\pm 1 \%$ ) (Figure 4F).

Brief ASO treatment does not induce neutrophil or monocyte activation

To further explore the interaction of ASO with mono- 
A

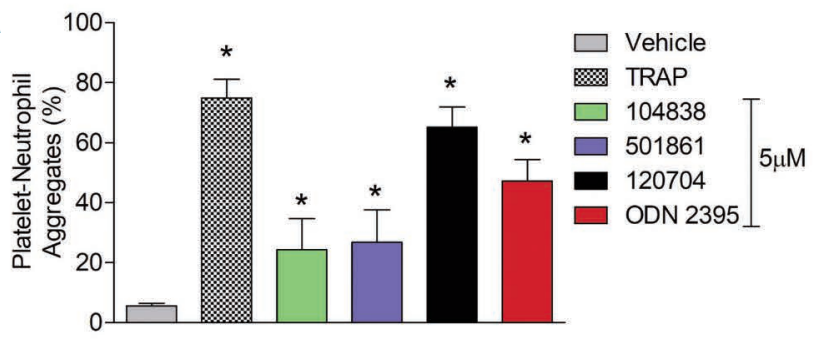

C

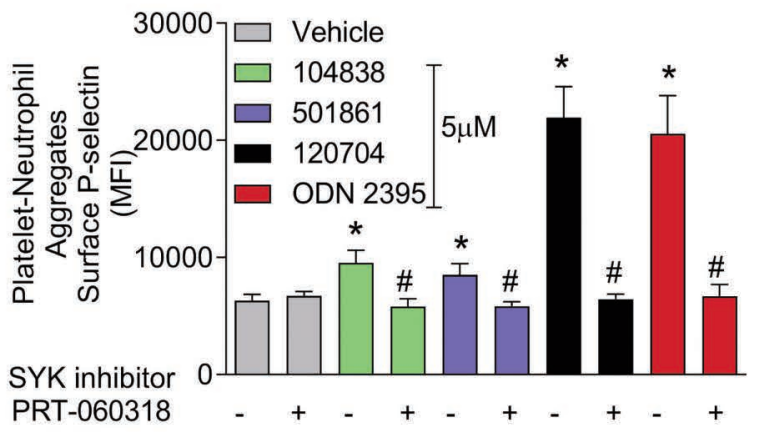

E

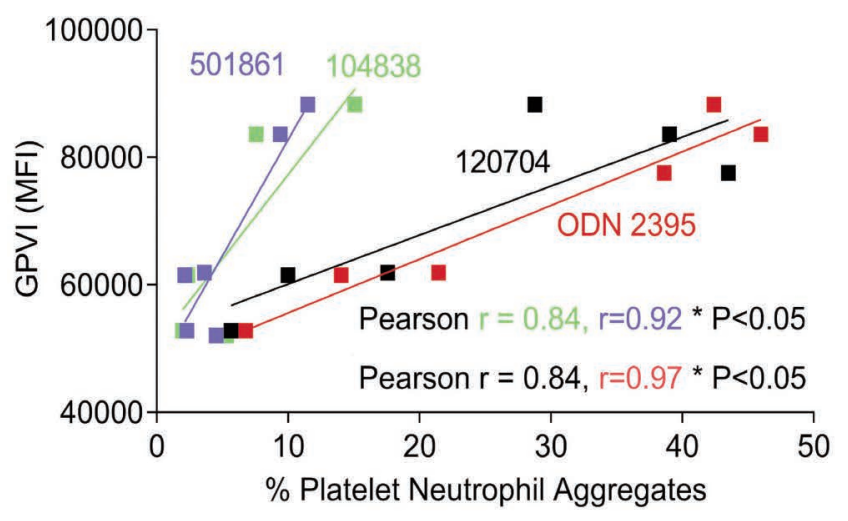

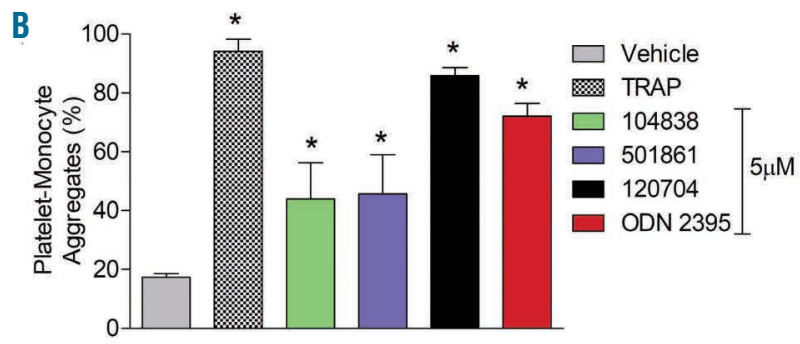

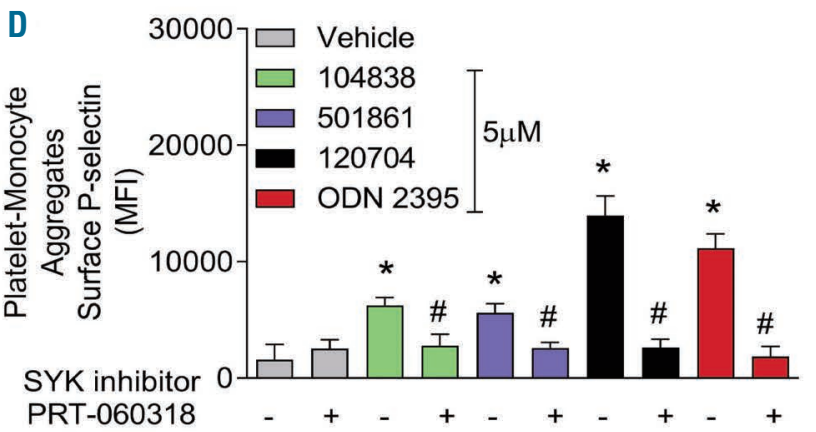

F

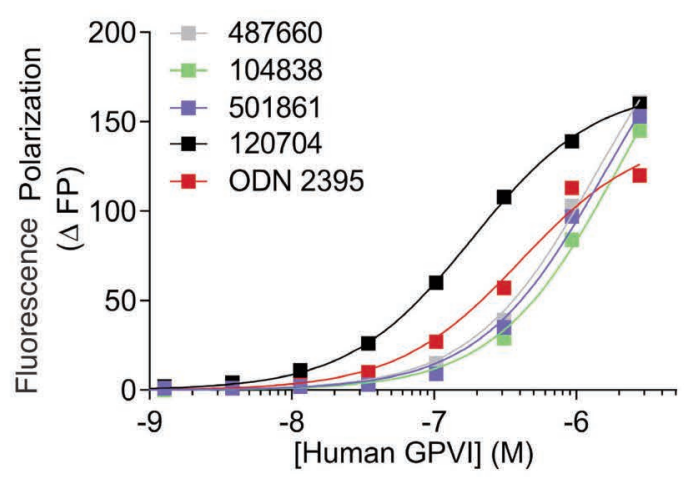

\begin{tabular}{|c|c|c|c|c|c|}
\hline Affinity to GPVI & 487660 & 104838 & 501861 & 120704 & ODN 2395 \\
\hline Bmax & 243 & 251 & 241 & 169 & 144 \\
\hline$K_{\mathrm{D}}[\mu \mathrm{M}]$ & 1.4 & 1.2 & 1.5 & 0.2 & 0.4 \\
\hline
\end{tabular}

Figure 7. The effect of antisense oligonucleotides on platelet-neutrophil, platelet-monocyte and glycoprotein VI interactions. Whole blood (WB) was incubated with vehicle (10 mM HEPES), thrombin receptor activating peptide (TRAP, $25 \mu \mathrm{M}$ ), or the 2'MOE antisense oligonucleotides (ASO): 104838 or 501861 , or the CpG ASO: 120704 or ODN 2395 (all at $5 \mu \mathrm{M}$ ) and analyzed by flow cytometry for (A) platelet-neutrophil aggregates (platelet marker CD41/61 ${ }^{+}$, neutrophil marker CD66 $\left.{ }^{+}\right)$, (B) platelet-monocyte aggregates (platelet marker CD41/61 ${ }^{+}$, monocyte marker CD14 ${ }^{+}$). (C) Surface P-selectin-positive (CD62 $\left.\mathrm{p}^{+}\right)$platelet-neutrophil aggregates $\left(\mathrm{CD} 41 / 61^{+}, \mathrm{CD}_{6} \mathrm{~b}^{+}, \mathrm{CD} 62 \mathrm{p}^{+}\right)$(median fluorescence intensity, MFI). (D) Surface P-selectin-positive platelet-monocyte aggregates (CD41/61 ${ }^{+}, \mathrm{CD} 14^{+}, \mathrm{CD}$ 2 $\left.\mathrm{p}^{+}\right)(\mathrm{MFI})$ in WB from five to nine human donors. (E) Individual donor platelet glycoprotein (GP)VI levels were correlated to platelet-neutrophil aggregate formation after treatment with the ASO in six human donors. ${ }^{*} P<0.05$ by Pearson correlation analysis. (F) A fluorescence polarization assay was used to measure binding affinity of Alexa647-labeled ASO to human GPVI. Bmax is the total density of receptors in a sample and $\mathrm{K}_{\mathrm{D}}$ is the equilibrium dissociation constant. The smaller the KD, the greater the binding affinity of the ASO to human GPVI.

cytes and neutrophils, we assessed whether ASO increased surface expression of CD11b, a broad immune cell activation marker. Lipopolysaccharide was included as a positive control to increase expression of CD11b on the surface of neutrophils (Online Supplementary Figure $S 4 A$ ) or monocytes (Online Supplementary Figure S4B). SYK is also involved in leukocyte intracellular signaling, ${ }^{27,28}$ and there was an inhibitory effect on CD11b surface expression in SYK-treated samples exposed to vehicle and lipopolysaccharide (Online Supplementary Figure S4 $A, B)$. None of the ASO tested had any effect on CD11b expression on either neutrophils or monocytes (after 30 min incubation); hence the ASO did not appear to activate these cells directly within this timeframe (Online
Supplementary Figure $S 4 A, B)$. In support of these data, the proinflammatory chemokines interleukin-8 (IL-8) and monocyte chemoattractant protein-1 (MCP-1) were not released from whole blood incubated with ASO (1, 5 and $10 \mu \mathrm{M}$ ) for $30 \mathrm{~min}$ (Figure 5A, B).

\section{Treatment with CpG ASO (but not 2'MOE ASO) leads to IL-8 and MCP-1 release}

CpG motifs have been shown to be immunostimulatory ${ }^{15}$ and proinflammatory effects of the CpG ASO 120704 and ODN 2395 were apparent after $6 \mathrm{~h}$ of incubation of whole blood, with 5 and $10 \mu \mathrm{M}$ (but not $1 \mu \mathrm{M}$ ) resulting in robust IL-8 and MCP-1 release (Figure 5C, D). None of the 2'MOE ASO evoked a proinflammatory effect at any 
of the concentrations (Figure 5C-D). The concentrationdependent effects of 120704 and ODN 2395 are shown in Figure 5E, F.

\section{8, 501861, 120704 and ODN 2395-induced formation of platelet-leukocyte aggregrates in unstimulated whole blood is blocked by SYK pretreatment}

When whole blood was pretreated with a SYK inhibitor, collagen-induced platelet-leukocyte aggregates were markedly reduced, while TRAP-induced platelet-leukocyte aggregates were maintained, confirming the selectivity of the SYK inhibitor (Figure 6A). SYK pre-treatment completely reversed the ASO-induced formation of platelet-leukocyte aggregates (Figure 5A). Confocal imaging of the aggregates using a leukocyte marker (CD45) and a platelet marker (CD41/61), confirmed the presence of platelet-leukocyte aggregates (Figure 6B) but showed greater platelet-platelet aggregation in the platelet-leukocyte aggregates in whole blood treated with the CpG ASO (which was more similar to TRAP-treated samples) compared to the 2'MOE ASO, which mainly involved single platelets bound to the leukocytes (Figure 6B).

\section{ASO increase levels of P-selectin-enriched platelet-neutrophil and platelet-monocyte aggregates, through a SYK-dependent mechanism, correlating to GPVI levels}

Further investigation into the types of immune cells that were driving the ASO-induced formation of plateletleukocyte aggregates revealed an increase in both platelet-neutrophil aggregates and platelet-monocyte aggregates (Figure 7A, B). The CpG ASO 120704 and ODN 2395 once again produced a more robust response than the 2'MOE ASO 104838 and 501861 (65 \pm 7 and 47 \pm 7 vs. $24 \pm 10$ and $27 \pm 11$ vs. $6 \pm 1$ for vehicle). The platelet-neutrophil and platelet-monocyte aggregates that formed in the ASO-treated samples were enriched in P-selectin (Figure 7C, D) and the greatest increase was observed in the samples treated with 120704 and ODN 2395. However, platelet-neutrophil aggregates and plateletmonocyte aggregates were not enriched in CD11b (Online Supplementary Figure S4C, D), implying that the initial formation of these aggregates was driven by activated platelets (not activated leukocytes). Pearson correlation analysis between platelet GPVI levels and platelet neutrophil aggregates showed a strong positive correlation for all the ASO tested; higher platelet GPVI expression was associated with a stronger platelet-neutrophil aggregate response (Figure $7 \mathrm{E}$ ).

\section{CpG ASO bind to GPVI with a higher affinity compared to 2'MOE ASO}

Fluorescence polarization experiments revealed that all ASO bound to human GPVI, with equilibrium dissociation constant $\left(\mathrm{K}_{\mathrm{D}}\right)$ values in the low micromolar range (Figure $7 \mathrm{~F}$ ): the smaller the $\mathrm{K}_{\mathrm{D}}$, the greater the binding affinity. All the ASO bound to GPVI with stronger affinity than to control human serum albumin (Online Supplementary Figure S5). The 2'MOE 487660 showed stronger affinity $\left(\mathrm{K}_{\mathrm{D}} 12.9 \mu \mathrm{M}\right)$ to human serum albumin than the other two 2'MOE ASO (104838 KD $24 \mu \mathrm{M}$ and $\left.501861 \mathrm{~K}_{\mathrm{D}} 26 \mu \mathrm{M}\right)$. The $\mathrm{CpG}$ ASO displayed higher affinity (lower $\mathrm{K}_{\mathrm{D}}$ ) for GPVI compared to the 2'MOE ASO (Figure 7F).

\section{Discussion}

The main findings from the current study are: (i) none of the ASO sequences investigated had an inhibitory effect on proplatelet production by either human- or mousederived megakaryocytes; (ii) all ASO demonstrated uptake into human platelets (2'MOE 487660 less than the others); (iii) a subset of 2'MOE ASO (104838 and 501861) and CpG ASO (120704 and ODN 2395) activated human platelets, triggering $\mathrm{P}$-selectin and SDF1 $\alpha$ release from platelet $\alpha$-granules and platelet-leukocyte aggregate formation; (iv) the ASO-induced platelet activation and platelet-leukocyte aggregate formation were fully reversed by pre-treatment with a SYK inhibitor; (v) ASO-induced platelet effects appeared to be sequence-dependent, rather than 2'MOE-dependent, since the 2'MOE ASO 487660 did not affect platelet function; (vi) all the ASO were shown to bind to human GPVI (CpG ASO had the strongest affinity); (vii) only the CpG ASO had a concentration-dependent proinflammatory effect, triggering IL-8 and MCP-1 release in whole blood after incubation for 6 h; (viii) the responsiveness to the ASO-induced plateletactivating and platelet-leukocyte aggregate-forming effects varied between donors and showed a strong positive correlation to individual platelet GPVI surface expression.

The novelty of this study concerns the effect of 2 ' $\mathrm{MOE}$ ASO on human platelets. Our focus was the 2 'MOE ASO 104838, which has been shown to be representative of a subset of ASO sequences that produce phenotype 1 reductions in platelet count in monkeys and humans. ${ }^{8,10} \mathrm{We}$ identified that the 2'MOE ASO 104838 binds to GPVI receptors on human platelets, increasing platelet surface P-selectin and prompting the formation of platelet-neutrophil aggregates and platelet-monocyte aggregates, most likely through an interaction between platelet P-selectin and leukocyte P-selectin glycoprotein ligand 1 (PSGL-1). ${ }^{29}$ This appears to be a platelet-driven interaction (at least when tested in vitro), as there were no signs of leukocyte activation (CD11b or IL-8/MCP-1 release) even after 10 $\mu \mathrm{M}$ of the 2 'MOE ASO 104838.

Mechanistically, we suggest that 2'MOE ASO 104838 lowers platelet count, producing a phenotype 1 , by activating platelets, an effect which triggers platelet-leukocyte aggregates and subsequent clearance of platelets by leukocytes. Maugeri et al. showed that platelet-neutrophil interactions might contribute to platelet clearance through active phagocytosis of $\mathrm{P}$-selectin-positive platelets by neutrophils. ${ }^{30}$ The internalization of platelets by neutrophils has been observed in patients with viral infection (associated with reductions in platelet count). ${ }^{31}$ Increased levels of P-selectin can also enhance sequestration of platelets on vascular endothelial surfaces. ${ }^{32}$ When monkeys were treated with 2'MOE ASO 104838, there was increased platelet sequestration in the densely vascularized liver and spleen. ${ }^{10}$ However, in vitro incubation of platelet-rich plasma from treatment-naïve monkeys with $2^{\prime} \mathrm{MOE}$ ASO 104838 did not lead to platelet activation ${ }^{10}$ (as we observed in human platelet-rich plasma and whole blood). This may be because the sample size used $(n=3)$ did not capture the variability seen in ASO responsiveness. Monkeys treated with 2'MOE ASO 104838 also had higher plasma levels of IL-8 and MCP-1,10 which we did not see in our in vitro assay with 104838 in human blood (using doses up to $10 \mu \mathrm{M}$ ). This may reflect in vitro rather than in 
vivo conditions or be due to species differences. Further investigation is warranted to see if in vivo treatment, or indeed clinical use of certain 2'MOE ASO sequences leads to increased platelet activation and formation of plateletleukocyte aggregates and to what degree this is paired with clinical reductions in platelet count.

Our data showing that pre-treatment with a SYK inhibitor was able to fully reverse ASO-induced platelet activation and platelet-leukocyte aggregate formation, speculatively highlights the potential of using a clinically available SYK inhibitor, for instance fostamatinib, ${ }^{33}$ to treat ASO-induced platelet side effects. This would need to be investigated further.

Flierl et al. were the first to identify that ODN 2395 binds to and activates GPVI on platelets. ${ }^{14}$ In the current study, we confirmed these findings and added the novel finding that 2'MOE ASO also bind to GPVI. Overall, the 2'MOE ASO had lower affinity to GPVI compared to the CpG ASO, which is consistent with their weaker platelet activating effects. In general it has been shown that the stronger affinity with which PS ASO bind, the larger the conformational change of the protein. ${ }^{34}$ Hence, CpG ASO, with their greater affinity than 2 'MOE ASO for GPVI, may induce a more substantial conformational change in the GPVI protein, affecting for instance GPVI clustering/dimerization, ${ }^{14}$ explaining the greater potency of $\mathrm{CpG}$ ASO to activate platelets.

Interestingly, the 2'MOE ASO 487660, which has not been shown to lower platelet counts in monkeys, did not affect any of the platelet function tests we performed in this study. Hence, the platelet effects observed with 104838 and 501861 appear to be sequence-dependent rather than due to their 2'MOE-backbone chemistry. 487660 did however still bind to human GPVI with a similar affinity as that of the other 2'MOE ASO, 104838 and 501861. This finding highlights the importance of functional in vitro platelet studies beyond ASO-GPVI binding assays in cell-free environments. The discrepancy by which 2'MOE ASO 487660 can bind GPVI but does not activate platelets may be explained by its greater affinity to bind human serum albumin, compared to the other 2'MOE ASO investigated, which may keep 487660 more bound to plasma proteins. 2'MOE ASO 487660 may also interact differently with the GPVI receptor as there appeared to be less surface and internalized 2'MOE ASO 487660 staining in platelets in the electron-microscopy images.
There is a degree of subject variability in platelet count reductions following ASO treatment in monkeys and humans..$^{8,10,16}$ We also noticed a high degree of variability in responsiveness to ASO in our in vitro studies of blood from healthy human donors. Although the sample was small $(n=7)$, there was a strong positive correlation between an individual's platelet GPVI levels and their platelet responsiveness to both 2'MOE (104838 and 501861) and CpG ASO-induced activation. Platelet GPVI levels have been shown to vary in healthy individuals ${ }^{35}$ and to be increased in different disease states such as obesity. ${ }^{36}$ Platelet GPVI levels could potentially be useful as a screening tool (used before commencement of treatment) to identify at-risk patients who may be more susceptible to platelet side effects of some sequence-specific GPVIactivating ASO.

We have shown that 2'MOE ASO that have been associated with phenotype 1 may not simply be reducing platelet counts, but can also have direct effects on platelets, triggering interactions between platelets and immune cells.

In summary, we have defined new mechanisms by which 2'MOE ASO-based drugs affect human platelets, which may yield new strategies to avoid ASO sequences with unfavorable platelet effects.

\section{Disclosures}

JEI has financial interest in and is a founder of Platelet BioGenesis, a company that aims to produce donor-independent human platelets from human-induced pluripotent stem cells at scale. The interests of JEI were reviewed and are managed by the Brigham and Women's Hospital and Partners HealthCare. The remaining authors declare that they have no conflicts of interest.

\section{Contributions}

MHLS supervised the study, performed research, analyzed data and wrote the manuscript; PV, IT, HR, GC, AW, HG, JM, $R O, B E$, and JT performed research, analyzed data and edited the manuscript; JWS, SPH and PN analyzed data and edited the manuscript; JEI supervised the study, analyzed data and edited the manuscript.

\section{Funding}

This work was funded by a corporate sponsored research agreement between Ionis Pharmaceuticals Inc. and Brigham and Women's Hospital and Partners HealthCare in accordance with their conflict-of-interest policies.

\section{References}

1. Bennett CF, Baker BF, Pham N, Swayze E, Geary RS. Pharmacology of antisense drugs. Annu Rev Pharmacol Toxicol. 2017:57:81-105.

2. Bennett CF, Swayze EE. RNA targeting therapeutics: molecular mechanisms of antisense oligonucleotides as a therapeutic platform. Annu Rev Pharmacol Toxicol. 2010;50:259-293.

3. Finkel RS, Mercuri E, Darras BT, et al. Nusinersen versus sham control in infantile-onset spinal muscular atrophy. N Engl J Med. 2017;377(18):1723-1732.

4. Mercuri E, Darras BT, Chiriboga CA, et al. Nusinersen versus sham control in lateronset spinal muscular atrophy. $\mathrm{N}$ Engl J Med. 2018;378(7):625-635.
5. Mathew V, Wang AK. Inotersen: new promise for the treatment of hereditary transthyretin amyloidosis. Drug Des Devel Ther. 2019;13:1515-1525.

6. Geary RS, Baker BF, Crooke ST. Clinical and preclinical pharmacokinetics and pharmacodynamics of mipomersen (Kynamro $\left.{ }^{\circledR}\right)$ : a second-generation antisense oligonucleotide inhibitor of apolipoprotein B. Clin Pharmacokinet. 2015;54(2):133-146.

7. Schoch KM, Miller TM. Antisense oligonucleotides: translation from mouse models to human neurodegenerative diseases. Neuron. 2017;94(6):1056-1070.

8. Crooke ST, Baker BF, Witztum JL, et al. The effects of 2'-O-methoxyethyl containing antisense oligonucleotides on platelets in human clinical trials. Nucleic Acid Ther.
2017:27(3):121-129.

9. Chi X, Gatti P, Papoian T. Safety of antisense oligonucleotide and siRNA-based therapeutics. Drug Discov Today. 2017; 22(5):823-833.

10. Narayanan P, Shen L, Curtis BR, et al. Investigation into the mechanism(s) that leads to platelet decreases in Cynomolgus monkeys during administration of ISIS 104838, a 2'-MOE-modified antisense oligonucleotide. Toxicol Sci. 2018;164(2): 613-626.

11. Henry SP, Narayanan P, Shen L, Bhanot S, Younis HS, Burel SA. Assessment of the effects of 2'-methoxyethyl antisense oligonucleotides on platelet count in Cynomolgus nonhuman primates. Nucleic Acid Ther. 2017;27(4):197-208.

12. Narayanan P, Curtis BR, Shen L, et al. 
Underlying immune disorder may predispose some transthyretin amyloidosis subjects to inotersen-mediated thrombocytopenia. Nucleic Acid Ther. 2020;30(2):94103.

13. Aster RH, Bougie DW. Drug-induced immune thrombocytopenia. N Engl J Med. 2007;357(6):580-587.

14. Flierl U, Nero TL, $\operatorname{Lim} B$, et al. Phosphorothioate backbone modifications of nucleotide-based drugs are potent platelet activators. J Exp Med. 2015; 212(2):129-137.

15. Sewing $S$, Roth $A B$, Winter $M$, et al. Assessing single-stranded oligonucleotide drug-induced effects in vitro reveals key risk factors for thrombocytopenia. PLoS One. 2017;12(11):e0187574.

16. Sewell KL, Geary RS, Baker BF, et al. Phase I trial of ISIS 104838, a 2'-methoxyethyl modified antisense oligonucleotide targeting tumor necrosis factor-alpha. J Pharmacol Exp Ther. 2002;303(3):13341343.

17. Ferrer-Marin F, Stanworth S, Josephson C, Sola-Visner M. Distinct differences in platelet production and function between neonates and adults: implications for platelet transfusion practice. Transfusion. 2013:53(11):2814-2821.

18. Vijey P, Posorske B, Machlus KR. In vitro culture of murine megakaryocytes from fetal liver-derived hematopoietic stem cells. Platelets. 2018;29(6):583-588

19. French SL, Vijey P, Karhohs KW, et al. High Content, Label-free analysis of proplatelet production from megakaryocytes. J Thromb Haemost. 2020;18(10):2701-2711.

20. Yu RZ, Grundy JS, Geary RS. Clinical pharmacokinetics of second generation antisense oligonucleotides. Expert Opin Drug
Metab Toxicol. 2013;9(2):169-182.

21. Thon JN, Devine MT, Jurak Begonja A, Tibbitts J, Italiano JE Jr. High-content livecell imaging assay used to establish mechanism of trastuzumab emtansine (T-DM1)-mediated inhibition of platelet production. Blood. 2012;120(10):1975-1984.

22. Machlus KR, Wu SK, Stumpo DJ, et al. Synthesis and dephosphorylation of MARCKS in the late stages of megakaryocyte maturation drive proplatelet formation. Blood. 2016;127(11):1468-1480.

23. Gaus HJ, Gupta R, Chappell AE, Ostergaard ME, Swayze EE, Seth PP. Characterization of the interactions of chemically-modified therapeutic nucleic acids with plasma proteins using a fluorescence polarization assay. Nucleic Acids Res. 2019;47(3):1110-1122.

24. Rayes J, Watson SP, Nieswandt B. Functional significance of the platelet immune receptors GPVI and CLEC-2. J Clin Invest. 2019;129(1):12-23.

25. Chan MV, Leadbeater PD, Watson SP, Warner TD. Not all light transmission aggregation assays are created equal: qualitative differences between light transmission and 96-well plate aggregometry. Platelets. 2018;29(7):686-689.

26. Russell-Smith NC, Flower RJ, Cardinal DC Measuring platelet and leucocyte aggregation/adhesion responses in very small volumes of whole blood. J Pharmacol Methods. 1981;6(4):315-333.

27. Berton G, Mocsai A, Lowell CA. Src and Syk kinases: key regulators of phagocytic cell activation. Trends Immunol. 2005 26(4):208-214.

28. Miller YI, Choi SH, Wiesner P, Bae YS. The SYK side of TLR4: signalling mechanisms in response to LPS and minimally oxidized
LDL. Br J Pharmacol. 2012;167(5):990-999.

29. Kappelmayer J, Nagy B, Jr. The Interaction of selectins and PSGL-1 as a key component in thrombus formation and cancer progression. Biomed Res Int. 2017;2017: 6138145.

30. Maugeri N, Rovere-Querini P, Evangelista $\mathrm{V}$, et al. Neutrophils phagocytose activated platelets in vivo: a phosphatidylserine, $\mathrm{P}$ selectin, and $\beta 2$ integrin-dependent cell clearance program. Blood. 2009;113(21) 5254-5265.

31. Koupenova M, Vitseva O, MacKay CR, et al. Platelet-TLR7 mediates host survival and platelet count during viral infection in the absence of platelet-dependent thrombosis. Blood. 2014;124(5):791-802.

32. Ed Rainger G, Chimen M, Harrison MI, et al. The role of platelets in the recruitment of leukocytes during vascular disease. Platelets. 2015;26(6):507-520

33. Connell NT, Berliner N. Fostamatinib for the treatment of chronic immune thrombocytopenia. Blood. 2019;133(19):2027-2030.

34. Crooke ST, Liang XH, Crooke RM, Baker $\mathrm{BF}$, Geary RS. Antisense drug discovery and development technology considered in a pharmacological context. Biochem Pharmacol. 2020;114196.

35. Furihata K, Clemetson KJ, Deguchi $\mathrm{H}$, Kunicki TJ. Variation in human platelet glycoprotein VI content modulates glycoprotein VI-specific prothrombinase activity. Arterioscler Thromb Vasc Biol. 2001; 21(11):1857-1863.

36. Barrachina MN, Sueiro AM, Izquierdo I, et al. GPVI surface expression and signalling pathway activation are increased in platelets from obese patients: elucidating potential anti-atherothrombotic targets in obesity. Atherosclerosis. 2019;281:62-70. 